\title{
Cerebral Amyloid Angiopathy with Cortical Subarachnoid Hemorrhage as a Mimic for Transient Ischemic Attack: A Case Report
}

\author{
Mazyar Hashemilar (ib) ${ }^{1,}$ and Nasrin Forghani (i) ${ }^{1}$ \\ ${ }^{1}$ Department of Neurology, Tabriz University of Medical Sciences, Tabriz, Iran \\ "Corresponding author: Department of Neurology, Tabriz University of Medical Sciences, Tabriz, Iran. Email: mhashemilar@yahoo.com
}

Received 2020 November 17; Revised 2021 May 08; Accepted 2021 May 15.

\begin{abstract}
Context: Given its cerebral amyloid angiopathy, subarachnoid hemorrhage might represent transient focal neurological episodes erroneously diagnosed as transient ischemic attacks. The earliest neuroimaging findings in emergency room brain computed tomography indicating subarachnoid hemorrhage in these patients might be very subtle and missed by the clinician.

Case Presentation: An 80-year-old man referred with transient focal neurological episodes, suggestive of transient ischemic attacks. In general, except for some cognitive dysfunctions, no remarkable point was noticed in his neurological examination. Nonenhanced brain-computer tomography and magnetic resonance imaging revealed evidence indicating slight convexity subarachnoid hemorrhage at the left frontal cortical region.

Conclusions: The transient focal neurological episodes uncommonly represent intracranial hemorrhage. Nevertheless, this clinical representation might occur in patients with subarachnoid hemorrhage due to cerebral amyloid angiopathy. In such cases, the neuroimaging findings play a major role in the differential diagnosis. The misdiagnosis of transient ischemic attacks in these cases might lead to the consumption of antiplatelet drugs and end in catastrophic hemorrhage and life-threatening complications. Close attention to patients' clinical findings and judicious use of further neuroimaging studies would help clinicians to avoid making such mistakes.
\end{abstract}

Keywords: Cerebral Amyloid Angiopathy, Magnetic Resonance Imaging, Subarachnoid Hemorrhage, Transient Ischemic Attack

\section{Context}

Cerebral amyloid angiopathy (CAA) is the leading cause of intracranial hemorrhage and cognitive impairment in the elderly. It may present as a transient focal neurological episode (TFNE); however, most clinicians are not familiar with such a condition, thereby raising misdiagnosis. This case report aimed to introduce a case with uncommon symptoms of probable CAA to explain its manifestations to avoid misdiagnosis and life-threatening complications.

\section{Case Presentation}

An 80-year-old man with some paroxysms of transient neurologic deficits, including slurred speech, right upper limb weakness, and numbness with possible face and leg sparing, referred to our outpatient clinic. It was difficult for him to describe the paroxysms. The episodes lasted for 10 - 15 minutes and had occurred at least 2 - 3 times a day during the last two days with no loss of consciousness or headaches. He had no vascular risk factors except for age and no history of taking antiplatelet or anticoagulation agents. During the last year, he was suffering from progressive memory loss, for which he was taking donepezil (generic) 10 milligrams daily. Given his episodic memory impairment, it was difficult for him to provide a reliable history and describe the attacks; therefore, his son, who had also witness the attacks, were also asked about his history. He reported no involuntary movement or abnormal posture during the attacks. He captured a video from one of such attacks, presenting only slurred speech, difficulty in moving the right hand, and numbness.

The vital signs were normal during the examination the next day, and the general physical examination was normal as well. Neurological examination revealed no remarkable point except for the MMSE score $(=23)$. Furthermore, routine laboratory tests, including complete blood count, metabolic panel, electrolytes, and coagulation panel, were normal. The standard awake electroencephalogram was normal. The non-enhanced brain com- 


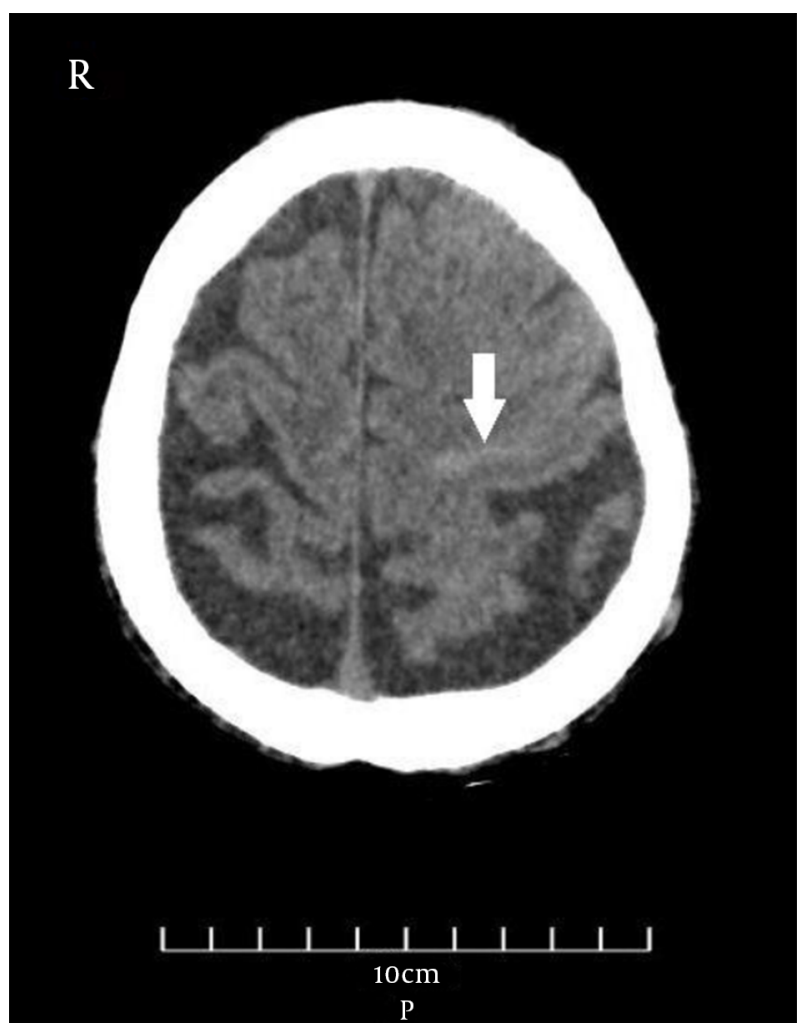

Figure 1. Non-enhanced computer tomography of the brain shows subtle SAH at the high left frontal convexity region (white arrow).

puted tomography (CT) scan showed an area of poorlydefined and fine hyperdensity in the sulci of the left frontal cortical region in agreement with convexity subarachnoid hemorrhage (CSAH) and a predominantly diffused cerebral cortical atrophy in bilateral temporal lobes (Figure 1). Brain magnetic resonance imaging (MRI) showed linear regions of blooming artifacts in accordance withblood products at the subarachnoid space of the left high frontal region on susceptibility-weighted image (SWI) sequences and hyperintense signals in the same region on fluidattenuated inversion recovery (FLAIR) sequences (Figures 2 and 3). The gadolinium-enhanced T1 image presented no abnormal enhancement, and no restriction on the diffusion weighted images of MRI was noticed. The brain and cervical magnetic resonance angiographies were normal. To control the attacks, which had made the patient and his family distressed and worried, oral carbamazepine (generic) $200 \mathrm{mg} /$ day was started and partially controlled them. The attacks stopped completely by a gradual increase of dosage up to $400 \mathrm{mg} /$ day in the following week.

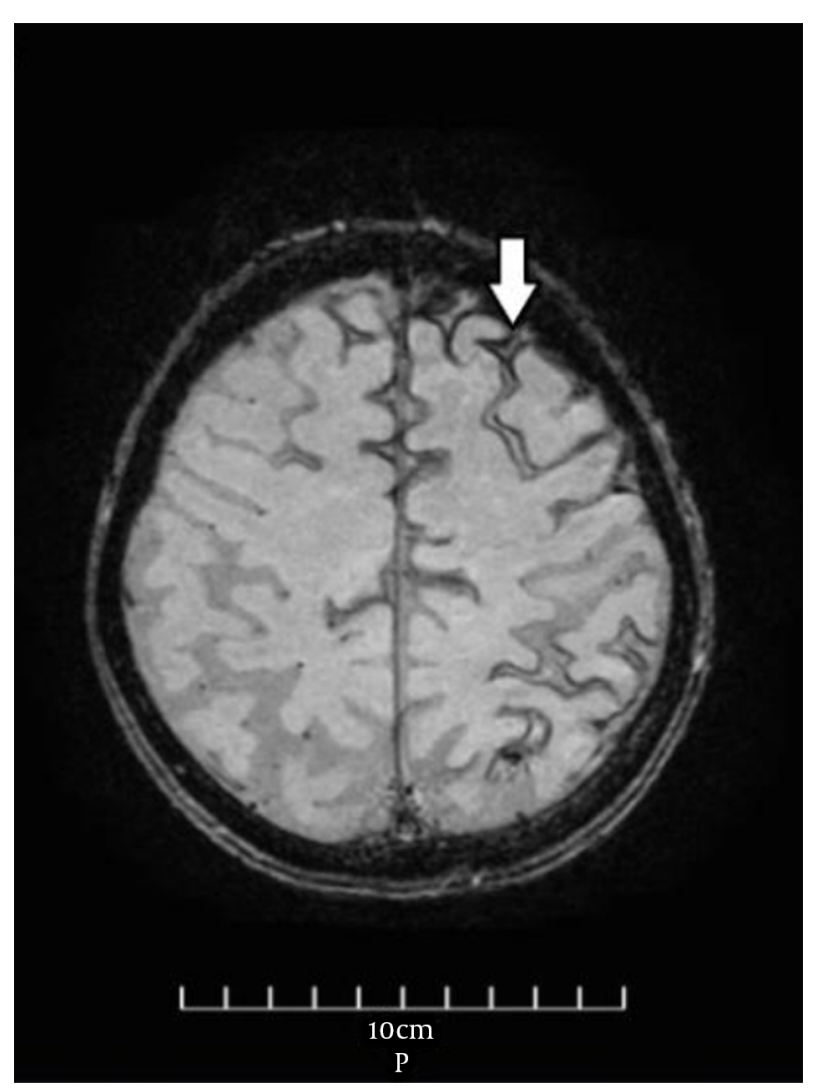

Figure 2. Brain MRI (susceptibility-weighted image sequence) shows linear bloom ing artifact (white arrow) in accordance with blood products in the subarachnoid spaces of the high left frontal convexity region.

\section{Discussion}

Cerebral amyloid angiopathy (CAA) is a small vessel disease of the brain in which beta-amyloid deposits in the small cortical and leptomeningeal arterioles. Cerebral amyloid angiopathy is a relatively common neuropathological abnormality and the etiology of lobar cerebral parenchymal hemorrhage and cognitive decline in the elderly (1). The clinical manifestations form a spectrum, including lobar intracerebral hemorrhage (ICH), transient focal neurologic episodes (TFNEs), also known as amyloid spells, cognitive impairment, and seizures $(2,3)$. Moreover, the neuroimaging features include lobar microbleeds on MRI, white matter hyperintensities, enlarged perivascular space in centrum semiovale, leukoencephalopathy, and cerebral atrophy (nonspecific finding due tochronic small vessel ischemia caused bybeta-amyloid deposition) (4-6). Other relevant imaging features are cortical superficial siderosis (CSS) on MRI and CSAH (7). Nontraumatic acute $\mathrm{CSAH}$ is limited to the subarachnoid space 


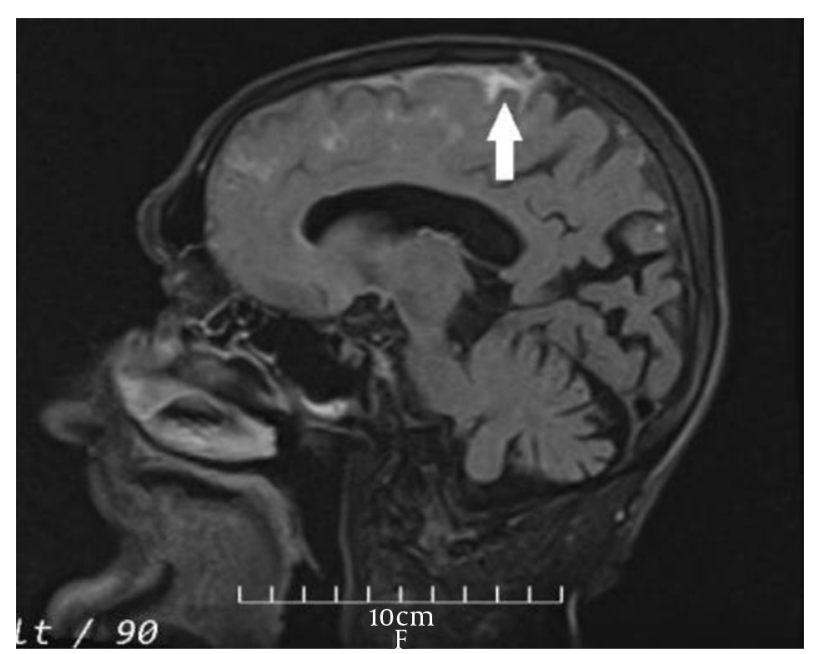

Figure 3. Brain MRI (FLAIR sequence) parasagittal plane image shows hyperintensity in the subarachnoid spaces of the high left frontal convexity region in agreement withhemorrhage (white arrow).

over the brain's cortical hemispheric convexities and does not involve cerebral parenchyma and ventricles. Different factors affect the emergence of cSAH. In a study on 742 cases, the most common factors leading to cSAH were as follows: CAA (39\%), reversible cerebral vasoconstriction syndrome (RCVS) (17\%), cerebral venous sinus thrombosis (10\%), large-vessel stenotic atherosclerosis (10\%), and posterior reversible encephalopathy syndrome (5\%). In this study, CAA and RCVS were the leading causes of cSAH in elderly patients aged above and below 60 years, respectively (6).

CAA may emerge with transient focal neurological deficits (TFNE) $(8,9)$; therefore, cSAH-induced TFNE can mimic transient ischemic attacks (TIA). Antiplatelet drugs are considered as a standard treatment for TIA. Avoiding anticoagulants and antiplatelet agents is generally recommended in the known CAA cases due to their potentials in inducing recurrent cerebral hemorrhagic complications (10). The wrong diagnosis of TIA in a previously unknown CAA case presented with TFNE may have catastrophic implications for the patient. This is further complicated by the subtlety of the imaging features, as in our case, regarding CSAH in the emergency brain CT scan, which are usually interpreted by non-expert physicians in emergency rooms. Both imaging and some clinical features help clinicians distinguish the symptoms in their diagnoses. The cSAH-induced TFNEs are associated with the most migratory symptoms, sensory disturbance, recurrent stereotyped events, and the lower prevalence of hypercholes- terolemia (9). In previous studies, lobar ICH is reported to be a strong predictor of re-bleeding; however, there is no sufficient data for re-bleeding following convexity SAH (8). As shown in our patient's imaging samples, a combination of FLAIR/SWI MR imaging can contribute to clarifying the diagnosis of suspected cSAH cases in a non-enhanced early brain CT scan (7). The definite diagnosis of CAA is possible only by complete postmortem histopathologic studies to confirm the deposition of beta-amyloid in the cerebral vasculature. However, with the introduction of the imagingbased Boston criteria in the diagnosis of CAA, it was possible to diagnose probable CAA in alive patients with no pathologic investigation of brain tissues. In this regard, the Boston criteria have recently been updated (11). According to the modified Boston criteria, our patient is a case of possible CAA. Cerebral amyloid angiopathy is a risk factor for dementia, and its symptoms mainly encompass perceptual speed, episodic and semantic memory deficits, and impaired attention (12). In our patient, the cognitive decline had started before the diagnosis of CAA. Some reports have suggested that TFNEs might be controlled in some cases by the antiepileptic treatment, as it was the case in our patient (13).

TFNEs are uncommon manifestations of cSAH caused by CAA. TFNEs, as a mimic of TIAs might complicate the course of the cSAH-induced CAA by the erroneous use of antithrombotic drugs, resulting in recurrent life-threatening intracerebral hemorrhage. A careful interpretation of neuroimaging and the use of specific brain MRI sequences such as SWI would guide clinicians in their diagnosis.

\section{Footnotes}

Authors' Contribution: Study concept and design: M. H.; Data collection: M. H. and N. F.; Drafting the manuscript: M. H. and N. F.; Critical revision of the manuscript: M. H.; Administrative, technical, and material support: M. H. and N.F; Study supervision: M. H.

Conflict of Interests: All authors declare that they have no potential conflict of interest.

Funding/Support: The authors declare that this study has received no financial support.

Informed Consent: Informed consent was obtained from the patient's son as his legal guardian.

\section{References}

1. Charidimou A, Boulouis G, Fotiadis P, Xiong L, Ayres AM, Schwab KM, et al. Acute convexity subarachnoid haemorrhage and cortical super- 
ficial siderosis in probable cerebral amyloid angiopathy without lobar haemorrhage. J Neurol Neurosurg Psychiatry. 2018;89(4):397-403. doi: 10.1136/jnnp-2017-316368. [PubMed: 29054916].

2. Eng JA, Frosch MP, Choi K, Rebeck GW, Greenberg SM. Clinical manifestations of cerebral amyloid angiopathy-related inflammation. Ann Neurol. 2004;55(2):250-6. doi: 10.1002/ana.10810. [PubMed: 14755729].

3. Reijmer YD, van Veluw SJ, Greenberg SM. Ischemic brain injury in cerebral amyloid angiopathy.J Cereb Blood Flow Metab. 2016;36(1):4054. doi: 10.1038/jcbfm.2015.88. [PubMed: 25944592]. [PubMed Central: PMC4758563].

4. Chao CP, Kotsenas AL, Broderick DF. Cerebral amyloid angiopathy: CT and MR imaging findings. Radiographics. 2006;26(5):1517-31. doi: 10.1148/rg.265055090. [PubMed: 16973779].

5. Chen SJ, Tsai HH, Tsai LK, Tang SC, Lee BC, Liu HM, et al. Advances in cerebral amyloid angiopathy imaging. Ther Adv Neurol Disord. 2019;12(1):1-11. doi: 10.1177/1756286419844113. [PubMed: 31105769]. [PubMed Central: PMC6501479].

6. Khurram A, Kleinig T, Leyden J. Clinical associations and causes of convexity subarachnoid hemorrhage. Stroke. 2014;45(4):1151-3. doi: 10.1161/STROKEAHA.113.004298. [PubMed: 24496391].

7. Verma RK, Kottke R, Andereggen L, Weisstanner C, Zubler C, Gralla J, et al. Detecting subarachnoid hemorrhage: comparison of combined FLAIR/SWI versus CT. Eur J Radiol. 2013;82(9):1539-45. doi: 10.1016/j.ejrad.2013.03.021. [PubMed: 23632159].

8. Wilson D, Hostettler IC, Ambler G, Banerjee G, Jager HR, Werring DJ. Convexity subarachnoid haemorrhage has a high risk of intrac- erebral haemorrhage in suspected cerebral amyloid angiopathy. $J$ Neurol. 2017;264(4):664-73. doi: 10.1007/s00415-017-8398-y. [PubMed: 28154972]. [PubMed Central: PMC5374182].

9. Stanton JED, Chandratheva A, Wilson D, Hostettler IC, Islam S, Werring DJ. Clinical features distinguish cerebral amyloid angiopathyassociated convexity subarachnoid haemorrhage from suspected TIA. J Neurol. 2020;267(1):133-7. doi: 10.1007/s00415-019-09558-9. [PubMed: 31583428]. [PubMed Central: PMC6954887].

10. Charidimou A, Shoamanesh A, Al-Shahi Salman R, Cordonnier C, Perry LA, Sheth KN, et al. Cerebral amyloid angiopathy, cerebral microbleeds and implications for anticoagulation decisions: The need for a balanced approach. Int J Stroke. 2018;13(2):117-20. doi: 10.1177/1747493017741384. [PubMed: 29125055].

11. Raposo N, Viguier A, Cuvinciuc V, Calviere L, Cognard C, Bonneville $\mathrm{F}$, et al. Cortical subarachnoid haemorrhage in the elderly: a recurrent event probably related to cerebral amyloid angiopathy. EurJ Neurol. 2011;18(4):597-603. doi: 10.1111/j.1468-1331.2010.03214.x. [PubMed: 21040231].

12. Planton M, Raposo N, Albucher JF, Pariente J. Cerebral amyloid angiopathy-related cognitive impairment: The search for a specific neuropsychological pattern. Rev Neurol (Paris). 2017;173(9):562-5. doi 10.1016/j.neurol.2017.09.006. [PubMed: 28993004].

13. Greenberg SM, Charidimou A. Diagnosis of cerebral amyloid angiopathy: Evolution of the boston criteria. Stroke. 2018;49(2):491-7. doi 10.1161/STROKEAHA.117.016990. [PubMed: 29335334]. [PubMed Central: PMC5892842]. 